\title{
Differential control of growth, cell cycle progression, and gene expression in human estrogen receptor positive MCF-7 breast cancer cells by extracts derived from polysaccharopeptide I'm-Yunity and Danshen and their combination
}

\author{
TZE-CHEN HSIEH and JOSEPH M. WU \\ Department of Biochemistry and Molecular Biology, New York Medical College, Valhalla, NY 10595, USA
}

Received April 19, 2006; Accepted June 12, 2006

\begin{abstract}
The use of herbs has been the mainstay of treatment for a variety of human illnesses and is an essential part of culturally-based healing traditions in many societies and countries. Also, herbs, including Chinese herbs, are being incorporated as remedies for disease management and treatment in Western countries. In Traditional Chinese Medicine (TCM), herbal prescriptions are most frequently given to patients as complex formulations containing multiple herbs. Notably and unwittingly, this approach amounts to the administration of several chemical entities at once; the underlying theory is that interactions among the chemicals present in different herbs in a formula exert synergistic pharmacodynamic actions and neutralize the adverse effects and toxicities of specific individual chemicals. The effectiveness and mechanisms of this approach have not been well investigated or understood. A primary interest of this laboratory is to obtain experimental evidence that supports the fundamental mechanistic theme for the combinatorial herbal strategy described above and its potential application in preventing and treating breast cancer $(\mathrm{BCa})$. In this study, we investigated the effects of $70 \%$ ethanolic extracts prepared from medicinal mushroom extract denoted I'm-Yunity and Danshen (Salvia miltiorrhizae Binge), alone and in combination, using MCF-7 cells as an in vitro model of estrogen receptor positive $\left(\mathrm{ER}^{+}\right)$, low invasive BCa. Combination of I'm-Yunity and Danshen (referred to as I'm-Yunity-Plus) suppressed clonogenicity to a comparable degree as Danshen alone; both being significantly more active than I'm-Yunity. However, extract of Danshen was more active in inhibiting MCF-7 cell growth
\end{abstract}

Correspondence to: Dr Joseph M. Wu, Room 147, Department of Biochemistry and Molecular Biology, Basic Sciences Building, New York Medical College, Valhalla, NY 10595, USA

E-mail: joseph_wu@nymc.edu

Key words: I'm-Yunity, Danshen, I'm-Yunity-Plus, cell cycle control, induction of apoptosis, breast carcinogenesis than I'm-Yunity-Plus. In comparison, I'm-Yunity elicited less growth inhibition. Flow cytometric analysis showed that I'm-Yunity-Plus induced partial block of $\mathrm{G}_{1} / \mathrm{S}$ transition in MCF-7 cells, whereas Danshen slowed down cell progression from $\mathrm{G}_{1} / \mathrm{S}$ into $\mathrm{G}_{2} / \mathrm{M}$ phases of the cell cycle. Treatment by I'm-Yunity did not affect cell cycle progression in MCF-7 cells; however, it promoted active induction of apoptosis. In addition, treatment with Danshen alone resulted in a pronounced reduction in the expression of $\mathrm{Rb}$, cyclin $\mathrm{D} 1$, and p53, and also led to a diminution of p65 and p50 forms of NF-кB. The pronounced suppressive effects of Danshen on expression of the aforementioned genes were largely attenuated in cells treated with I'm-Yunity-Plus suggesting that ingredients in Danshen must have interacted with those in I'm-Yunity as to culminate in neutralization of the gene suppressive effects of Danshen. Additional support for such interactions was obtained by targeted cDNA array analysis using human tumor metastasis and $\mathrm{BCa} / \mathrm{ER}$ signaling gene arrays. Taken together, our results are consistent with the interpretation that interaction exists between Danshen and I'm-Yunity and that I'm-Yunity-Plus may have efficacy in the treatment of BCa, particularly for patients with $\mathrm{ER}^{+}$status.

\section{Introduction}

Breast cancer $(\mathrm{BCa})$ is the most common cancer among women in the US and other developed and also developing countries $(1,2)$. There is consensus among clinicians and researchers that $\mathrm{BCa}$ is a preventable disease. Combination chemotherapies produce more clinically relevant, longer duration, better survival responses than single agent therapies and hence, have become the standard of management for metastatic, hormone-refractory $\mathrm{BCa}$, and increasingly, also primary $\mathrm{BCa}$. Unfortunately, they also produce significant side effects and long-term treatment can further be complicated by development of chemoresist tumor clones (3). Consequently, individuals diagnosed with $\mathrm{BCa}$ seek alternative treatment modalities including complementary and alternative medicine (CAM), to bolster the primary means of medical intervention and to alleviate their adverse effects. It has been estimated that as many as $50 \%$ of breast cancer patients use CAM, particularly biopharmacologic and herbal approaches (4). 
Herbal medicine is one of the most ancient forms of health care known to humankind and has been used in many cultures throughout history (5-8). Typically, herbal remedies emphasize the use of whole extracts from a single herb or combined extracts from multiple herbs in the form of formulations, as is the practice among Chinese herbalists and Traditional Chinese Medicine (TCM) specialists $(9,10)$. This method of disease management and treatment inadvertently enforces the administration of several chemical entities at once; the underlying theory is that interactions among the chemicals present in different herbs in a formulation likely will exert synergistic pharmacodynamic actions while simultaneously neutralize the adverse effects and toxicities of specific individual chemicals (11-13). In certain respects, therefore, herbal remedies resemble some of the same fundamental themes of chemoprevention, a concept originally introduced by Sporn in the 1970s advocating the use of natural or synthetic compounds to inhibit or retard cancer development (14-17). A major deficiency of the polyherbal formulation approach is that the effectiveness and mechanisms have not been well investigated or understood.

We sought to obtain experimental evidence that supports the fundamental mechanistic theme for the combinatorial herbal strategy described above and its potential application in preventing and treating $\mathrm{BCa}$. In the present study, we investigated the effects of $70 \%$ ethanolic extracts prepared from medicinal mushroom extract denoted I'm-Yunity and Danshen (Salvia miltiorrhizae Binge), alone and in combination (combination of I'm-Yunity and Danshen is referred to as I'm-Yunity-Plus), using MCF-7 cells as an in vitro model of estrogen receptor positive $\left(\mathrm{ER}^{+}\right)$, minimally invasive $\mathrm{BCa}$. I'm-Yunity is a family of distinct, heterogeneous polysaccharide-protein complexes isolated from cultivated mycelia of the Basidomycetes mushroom, Trametes (formerly Coriolus) versicolor, that have been used in many traditional Asian formulas to prevent or treat various types of cancer as well as to treat or prevent manifestations of enteric inflammation (18-21). Danshen (Salvia miltiorrhizae), which historically, is the TCM chosen for treating circulatory disorders, have also been used in treating certain cancers and as chemopreventive agents $(22,23)$. I'm-Yunity-Plus suppressed clonogenicity to a degree comparable to the effects of adding Danshen alone, and both were more active than cells treated with I'm-Yunity. However, extract of Danshen was more active in inhibiting MCF-7 cell growth than I'm-Yunity-Plus. In comparison, I'm-Yunity showed less growth inhibition. Flow cytometric analysis showed that I'm-Yunity-Plus induced partial block of $G_{1} / S$ transition in MCF-7 cells, whereas Danshen slowed down cell progression from $G_{1} / S$ into $G_{2} / M$ phases of the cell cycle. I'm-Yunity, however, only actively induced apoptosis in MCF-7 cells without affecting cell cycle progression. In addition, only cells treated with Danshen resulted in a pronounced reduction in the expression of $\mathrm{Rb}$, cyclin $\mathrm{D} 1$, and $\mathrm{p} 53$, as well as diminished levels of $\mathrm{p} 65$ and $\mathrm{p} 50$ forms of $\mathrm{NF}-\mathrm{\kappa B}$. The pronounced suppressive effects of Danshen on expression of the aforementioned genes were largely attenuated in cells identically treated with I'm-Yunity-Plus suggesting that there must have been interactions between ingredients in Danshen with those in I'm-Yunity, the molecular outcome of which is the neutralization of the gene suppressive effects of Danshen. Support for such interactions was also evident using cDNA array analyses targeting tumor metastasis and $\mathrm{BCa} / \mathrm{ER}$ signaling genes.

Taken together, our results are consistent with the interpretation that interaction exists between Danshen and I'm-Yunity and that I'm-Yunity-Plus may be considered as complementary therapy for patients diagnosed with $\mathrm{BCa}$, particularly individuals with $\mathrm{ER}^{+}$status.

\section{Materials and methods}

Cell cultures. The breast adenocarcinoma cell line MCF-7 was purchased from American Type Culture Conditions (ATCC, Rockville, MD). Cells were maintained in Eagle's minimum essential medium supplemented with $2 \mathrm{mM}$ glutamine and Earle's BSS adjusted to contain $1.5 \mathrm{~g} / 1$ sodium bicarbonate, $0.1 \mathrm{mM}$ non-essential amino acids and $1 \mathrm{mM}$ sodium pyruvate and supplemented with $0.01 \mathrm{mg} / \mathrm{ml}$ bovine insulin and $10 \%$ fetal bovine serum, as described (6). Cells were seeded at a density of $5 \times 10^{4}$ cells $/ \mathrm{ml}$ and passaged by washing the mono-layers with phosphate-buffered saline (PBS) followed by a brief incubation with $0.25 \%$ trypsin or trypsin/EDTA. The washed cells were isolated by centrifugation and resuspended in culture medium for plating or counting.

Preparation of ethanolic extracts of I'm-Yunity, Danshen and I'm-Yunity-Plus. I'm-Yunity, Danshen, and I'm-Yunity-Plus were provided as capsules or powder by Integrative Chinese Medicine Holdings Ltd. (TST East Kowloon, Hong Kong) and stored at $4{ }^{\circ} \mathrm{C}$. To prepare $70 \%$ ethanolic extracts, contents of each capsule of I'm-Yunity and I'm-Yunity-Plus were suspended in $70 \%$ ethanol (340 gram $/ 3.3 \mathrm{ml})$, followed by stirring the suspension at room temperature with intermittent mixing at $150 \mathrm{rpm}$ for $60 \mathrm{~min}$. Since each capsule of I'mYunity-Plus contained $110 \mathrm{mg}$ of Danshen, extracts of Danshen were prepared by suspending $110 \mathrm{mg}$ of Danshen in $3.3 \mathrm{ml}$ of $70 \%$ ethanol. For each preparation, the insoluble material was removed by centrifugation in a micro-centrifuge and the soluble supernatant was sterilized by passing through a $0.22 \mu \mathrm{M}$ filter and kept in aliquots at $4^{\circ} \mathrm{C}$. Before use, the stock was further diluted in tissue culture media to give the final indicated concentrations.

Effect of ethanol extracts of I'm-Yunity, I'm-Yunity-plus and Danshen on colony formation and cell proliferation in MCF-7 cells. Clonogenicity was assayed as described $(6,12)$. MCF-7 cells at 200 cells/ml RPMI-1640 and 10\% FBS were dispensed into individual wells of a 6-well tissue culture dish. Cultures received various doses of ethanol extracts of I'm-Yunity, I'mYunity-Plus or Danshen. Control cultures received equivalent amounts of $70 \%$ ethanol. After 14 days in culture the cells were fixed and stained with $0.1 \%$ crystal violet to visualize colonies for counting. The experiments were performed in duplicate or triplicate.

To determine effects of ethanolic extracts of I'm-Yunity, I'm-Yunity-Plus or Danshen on inhibition of cell growth, confluent MCF-7 cells were harvested by trypsinization and adjusted to a density of $5 \times 10^{4}$ cells $/ \mathrm{ml}$. For each treatment, 


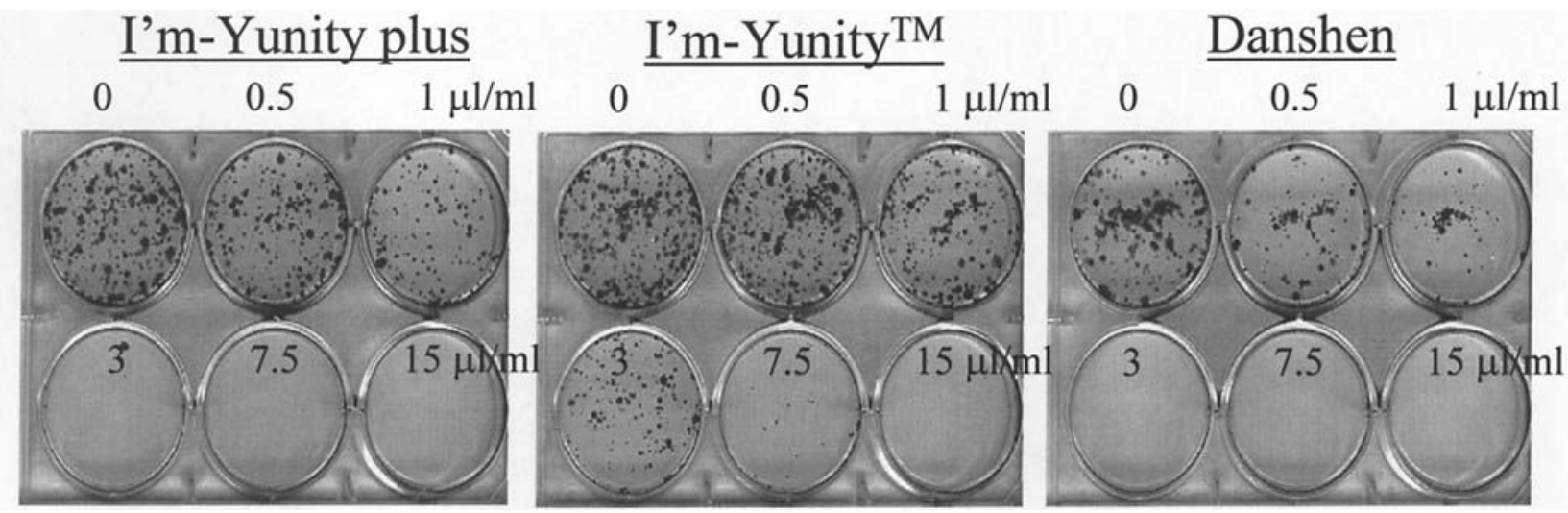

Figure 1. Effects of ethanolic extracts of I'm-Yunity, I'm-Yunity-plus, or Danshen on clonogenicity in MCF-7 cells. Clonogenicity was assayed as detailed in Materials and methods.

$10 \mathrm{ml}$ of cells were placed in T-75 flasks, together with $7.5 \mu \mathrm{l} /$ $\mathrm{ml}$ of $70 \%$ ethanol extracts of I'm-Yunity, I'm-Yunity-plus, Danshen, or the carrier solvent. Cells were harvested by trypsinization on days 1-3. Cell number in control and treated cells was counted using hemocytometer and cell viability was determined by trypan blue dye exclusion (24-26).

Effect of I'm-Yunity, Danshen and I'm-Yunity-Plus on induction of apoptosis and cell cycle progression. Cell cycle phase distribution was analyzed by flow cytometry. Cultures seeded at a density of $5 \times 10^{4}$ cells $/ \mathrm{ml}$ were incubated with $7.5 \mu 1 / \mathrm{ml}$ of $70 \%$ ethanol extracts of I'm-Yunity, I'm-Yunity-plus, Danshen, or the carrier solvent for 3 days and harvested. Cells were washed once with PBS and stained with $1.0 \mu \mathrm{g} / \mathrm{ml}$ 4,6-diamidion-2-phenylindole (DAPI; Molecular Probes, Eugene, OR) in a solution containing $100 \mathrm{mM} \mathrm{NaCl}, 2 \mathrm{mM}$ $\mathrm{MgCl}_{2}$ and $0.1 \%$ Triton X-100, $\mathrm{pH} 6.8$ as described (27-29). The DNA-specific DAPI fluorescence was excited with UV light and collected with appropriate filters in an ICP-22 flow cytometer (Ortho Diagnostic, Westwood, MA). The cell cycle distribution and percentage of apoptotic cells were analyzed by deconvoluting the DNA content frequency histograms with the use of CellFit software (Phoenix Flow, San Diego, CA), as detailed previously $(30,31)$.

cDNA array analysis. Total RNA was isolated from day 2 control and MCF-7 cells treated respectively with $7.5 \mu \mathrm{l} / \mathrm{ml}$ ethanol extracts of I'm-Yunity, I'm-Yunity-plus, Danshen, or the carrier solvent. RNA ( $4 \mu \mathrm{g})$ was used as the template for biotinylated cDNA synthesis. Hybridization of biotinylated cDNA to immobilized gene-specific cDNAs and detection of hybridization signals by chemiluminescence were according to the manufacturer's protocol (Superarray, Bethesda, MD). Briefly, the steps involved were: 1) prehybridization of membranes in solution containing $100 \mu \mathrm{g} / \mathrm{ml}$ heat-denatured sheared salmon sperm DNA, $2 \mathrm{~h}, 60^{\circ} \mathrm{C} ; 2$ ) overnight hybridization of membrane with the denatured cDNA probe, with continuous shaking at $10 \mathrm{rpm} / \mathrm{min}, 60^{\circ} \mathrm{C}$; 3) washing of membrane with pre-warmed 2X SSC and $1 \%$ SDS, followed by second washing with $0.1 \mathrm{X}$ SSC and $0.5 \%$ SDS; 4) blocking of membrane, 40 min using blocking solution $\mathrm{Q}$ provided by the manufacturer; 5) incubation of membrane with 1:7500 AP-streptavidin, $10 \mathrm{~min}$, room temperature; 6) incubation of washed and rinsed membrane with $1.0 \mathrm{ml} \mathrm{CDP-Star}$ chemi- luminescent substrate, followed by exposure to X-ray film. Routinely, multiple exposures were obtained to have more accurate and quantitative determination of the data. GEArray analyzed software was used for the data analysis.

Protein extraction and Western blot analysis. Control MCF-7 cells and cells treated with $7.5 \mu 1 / \mathrm{ml}$ ethanol extracts of I'mYunity, I'm-Yunity-plus, Danshen, or the carrier solvent were rinsed with ice-cold PBS and suspended in buffer (50 $\mu 1 / 10^{6}$ cells) containing $10 \mathrm{mM}$ Hepes, $\mathrm{pH} 7.5,90 \mathrm{mM} \mathrm{KCl}, 1.5 \mathrm{mM}$ $\mathrm{Mg}(\mathrm{OAc})_{2}, 1 \mathrm{mM}$ dithiothreitol, $0.5 \% \mathrm{NP} 40$ and 5\% glycerol supplemented with $0.5 \mathrm{mM}$ PMSF, $10 \mu \mathrm{g} / \mathrm{ml}$ each of aprotinin, pepstatin, leupeptin, and lysed by 3 freeze/thaw cycles $(6,26,30,31)$. The extracts were centrifuged and the clear supernatants were stored in aliquots at $-70^{\circ} \mathrm{C}$. Protein concentrations were measured with protein assay reagent (Pierce Chem. Co., Rockford, IL). For Western blot analysis, $10 \mu \mathrm{g}$ proteins were boiled for 5 min in Laemmli buffer and separated by $10 \%$ SDS-polyacrylamide gel electrophoresis (SDS-PAGE). The gels were transferred to nitrocellulose membranes by a semi-dry transfer method. After blocking with buffer containing 5\% low-fat milk, the membranes were probed for the expression of Rb, cyclins D1, p53, p21, NF-кB p65 and p50, and B-actin (Santa Cruz Biotechnology, Santa Cruz, CA). All antibodies used in the experiments were diluted at 1:1000. Specific immunoreactivity was demonstrated by enhanced chemiluminescence (ECL) or color reaction using procedures detailed in the manufacturer's protocol (Kirkegared \& Perry Laboratories, Gaithersburg, MD).

\section{Results}

Effects of ethanol extracts of I'm-Yunity, I'm-Yunity-plus, and Danshen on colony formation in MCF-7 cells. We first determined the effects of I'm-Yunity, I'm-Yunity-plus, and Danshen on colony formation, also referred to as clonogenicity, in MCF-7 cellls. This assay measures the ability of tumor cells to grow and form foci in a manner unrestricted by growth contact inhibition as is characteristically found in normal, untransformed cells. As such, clonogenicity provides an indirect assessment of the propensity of tumor cells to undergo neoplastic transformation. To measure clonogenicity, MCF-7 cells at a given cell density were plated onto multiple well tissue culture dishes, with and without addition of varying 

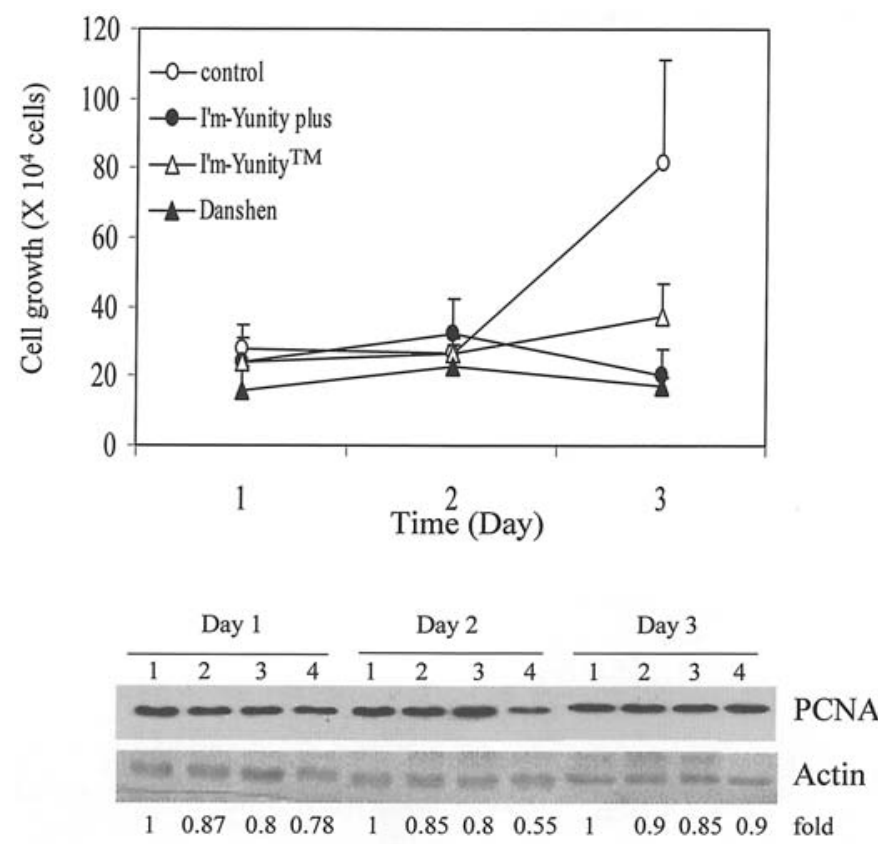

Figure 2. Time-dependent growth inhibition and PCNA expression in control and MCF-7 cells treated with $7.5 \mu \mathrm{l} / \mathrm{ml}$ ethanolic extracts of I'mYunity, I'm-Yunity-plus, or Danshen.

doses of $70 \%$ ethanolic extracts of I'm-Yunity, I'm-Yunity-plus, and Danshen. Control and treated cells were maintained in culture for an additional 14 days to allow formation of colonies. Size and number of colonies were visually inspected by fixing and staining in $0.1 \%$ crystal violet. Fig. 1 shows that clonogenicity of MCF-7 cells was significantly reduced by incubation with ethanol extracts of I'm-Yunity-plus and Danshen, with both showing a pronounced inhibition that was clearly evident at $\geq 1-3 \mu 1$. Notably, not only did Danshen reduced the number of colonies formed to a degree similar to treatment with I'm-Yunity-plus, it also visibly modulated the size of colonies formed, decreasing the sizes of some while stimulating those of others. Comparatively, $70 \%$ ethanolic extracts of I'm-Yunity were significantly less active in this assay, both with respect to the number and size of colonies formed as evident by the observation that inhibition became visually apparent only when the doses reached 7.5-15.0 $\mu 1$ (Fig. 1).

Effects of ethanol extracts of I'm-Yunity, I'm-Yunity-plus, and Danshen on growth of MCF-7 cells. We next tested the effects of I'm-Yunity, I'm-Yunity-plus, and Danshen on growth of MCF-7 cells. Results in Fig. 2A showed effects of $7.5 \mu \mathrm{l} /$ $\mathrm{ml}$ of ethanol extracts of I'm-Yunity, I'm-Yunity-plus, and Danshen. Significant reduction in cell growth was observed in cells treated with I'm-Yunity-plus and Danshen, while the inhibitory effects of I'm-Yunity were noticeably less prominent (panel A, Fig. 2). The suppression of cell proliferation by Danshen was accompanied by a transient reduction in expression of PCNA on day 1 , which became progressively more pronounced on day 2 and returned to control levels on day 3 of treatment. Neither I'm-Yunity or I'm-Yunity-plus suppressed PCNA expression.

\begin{tabular}{|l|c|c|c|c|c|}
\hline $\begin{array}{c}\text { Treatment } \\
(3 \text { days })\end{array}$ & $\begin{array}{c}\text { Dose } \\
(\mu \mathbf{l} / \mathrm{ml})\end{array}$ & \multicolumn{5}{|c|}{ Cell cycle distribution (\%) } \\
\hline & & G1 & S & G2/M & Apoptosis \\
\hline control & 0 & 48.2 & 36.8 & 15.1 & \\
\hline I'm-Yunity plus & 7.5 & 57.2 & 29.8 & 13.0 & 5.4 \\
\hline I'm-Yunity & 7.5 & 50.9 & 34.7 & 14.5 & 21.2 \\
\hline Danshen & 7.5 & 57.4 & 34.4 & 8.3 & \\
\hline
\end{tabular}

Figure 3. Cellular DNA content frequency histograms of MCF-7 cells showing the cell cycle phase distribution and apoptosis following a 3-day treatment with $7.5 \mu \mathrm{l} / \mathrm{ml}$ ethanolic extracts of I'm-Yunity, I'm-Yunity-plus, or Danshen. Flow cytometric analysis was performed as described in Materials and methods. Proportion of cells with fractional DNA content (sub- $\mathrm{G}_{1}$ cell population) was used to calculate induction of apoptosis resulting from treatment.

Effects of ethanol extracts of I'm-Yunity, I'm-Yunity-plus, and Danshen on cell cycle progression in MCF-7 cells. To explore the underlying basis for the antiproliferative activities of I'mYunity, I'm-Yunity-plus, and Danshen, cell cycle analyses were performed in MCF-7 cells, following a 3-day treatment with $7.5 \mu \mathrm{l} / \mathrm{ml}$ of ethanolic extracts. Cell cycle phase distribution was analyzed by flow cytometry. Control and treated cultures were harvested, washed with PBS and stained with $1.0 \mu \mathrm{g} / \mathrm{ml}$ DAPI, and analyzed as detailed in Materials and methods $(27,31)$. Results in Fig. 3 indicate that ethanol extracts of I'mYunity-Plus induced partial block of $\mathrm{G}_{1} / \mathrm{S}$ transition in MCF-7 cells, whereas Danshen slowed down cell progression from $\mathrm{G}_{1} / \mathrm{S}$ into $\mathrm{G}_{2} / \mathrm{M}$ phases of the cell cycle. By comparison, I'mYunity had virtually no effect on cell cycle progression but actively induced apoptosis in MCF-7 cells.

Modulation of cell growth regulatory protein expression by of ethanol extracts of I'm-Yunity, I'm-Yunity-plus, and Danshen in MCF-7 cells. Further evidence that these three herbal extracts elicited different cell cycle effects in MCF-7 cells came from analysis of changes in expression of proteins with key role in regulating cell cycle progression. For instance, MCF-7 cells treated with Danshen alone showed greatly suppressed expression of cyclin D1, whereas identical treatment with I'm-Yunity and I'm-Yunity-Plus had virtually no effect (Fig. 4). We also analyzed for changes in the retinoblastoma tumor suppressor protein $\mathrm{Rb}$, whose expression and state of phosphorylation play a pivotal role in the control of $\mathrm{G}_{1} / \mathrm{S}$ cell cycle checkpoint (28). Western blot analysis demonstrated that treatment with $7.5 \mu 1 / \mathrm{ml}$ ethanolic extract of Danshen significantly reduced the overall Rb levels, in MCF-7 cells (Fig. 4). Similarly, expression of p53 was down-regulated by ethanol extracts of Danshen but were unaffected by treatment with I'm-Yunity and I'm-Yunity-Plus.

The changes in cyclin D1 may explain in part the observed effect of Danshen on suppression of $\mathrm{S} / \mathrm{G}_{1}$ in MCF-7 cells but cannot easily support the apoptosis inducing effects of I'mYunity in MCF-7 cells. Accordingly, we measured changes in the steady state level of transcription factor NF-кB, known to be functionally associated with cell survival $(32,33)$. However, once again, only treatment with ethanolic extract of Danshen elicited a pronounced decrease in p65 and p50 forms of NF$\kappa \mathrm{B}$ in MCF-7 cells (Fig. 5). 


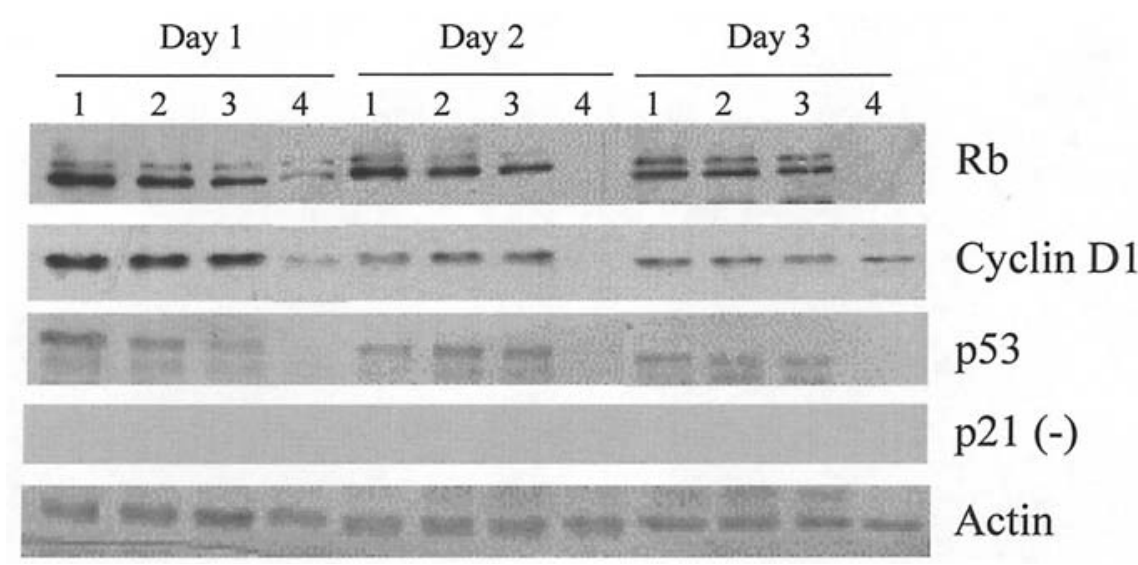

Figure 4. Expression of Rb, cyclin D1, p53 and p21 expression was investigated by immunoblot analysis as detailed in Materials and methods in MCF-7 cells treated for 1-3 days with $7.5 \mu \mathrm{l} / \mathrm{ml}$ ethanolic extracts of I'm-Yunity, I'm-Yunity-plus, or Danshen. A significant down-regulation on Rb, cyclin D1 and p53 was found in cells treated with Danshen.

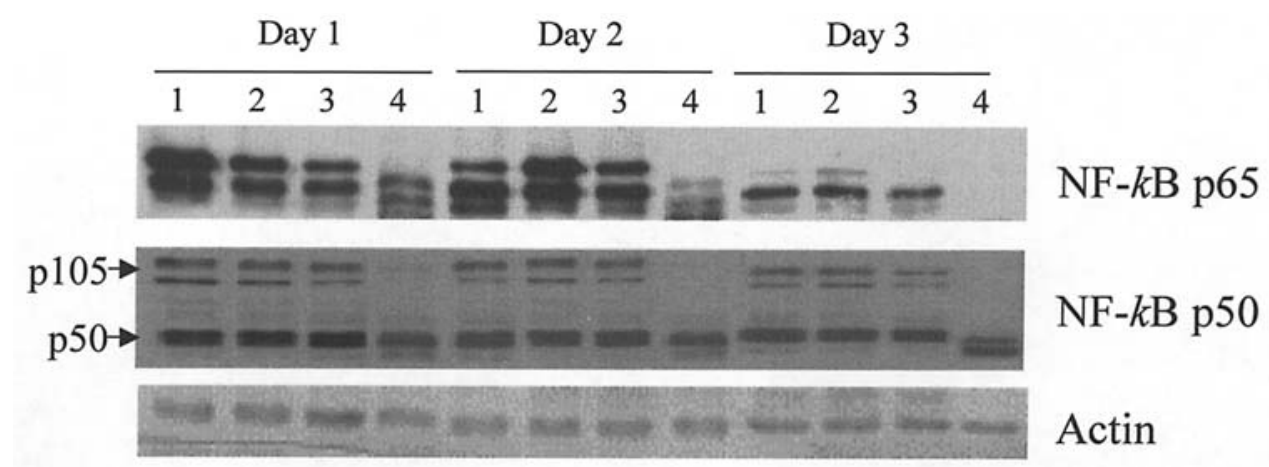

Figure 5. Expression of p65 and p50 forms of NFkB was investigated by immunoblot analysis as detailed in Materials and methods in MCF-7 cells treated for 1-3 days with $7.5 \mu 1 / \mathrm{ml}$ ethanolic extracts of I'm-Yunity, I'm-Yunity-plus, or Danshen. A significant down-regulation on NFkB was found in cells treated with Danshen.

Effects of ethanol extracts of I'm-Yunity, I'm-Yunity-plus, and Danshen on expression of tumor metastasis gene expression using cDNA array analysis. Human tumor metastasis cDNA array was used to detect changes in expression of a panel of cycle regulatory genes, in response to treatment with ethanol extracts of I'm-Yunity, I'm-Yunity-plus, and Danshen. This array contains genes that encode for several classes of protein factors including growth factors and their receptors, ECM components, proteases and their inhibitors, oncogenes and suppressors, and other related genes. This array can provide some insights into the molecular mechanism of metastasis or determine how a tumor metastasize. Through a side-by-side hybridization using experimental RNA samples and the arrays and reagents provided in the kit, it would be possible to simultaneously determine the expression profile of these genes involved in metastasis.

In these experiments, cells treated for 2 days were used in the analysis. Only integrin a6 showed a consistent up-regulation in response to treatment by all three herbal extracts. Expression of MMP15 was significantly increased by treatment with I'mYunity and was marginally increased by treatment with Danshen. Interestingly, MMP15 was significantly suppressed by I'm-Yunity-Plus. In addition, a number of other genes, notably, caveolin, c-myc and ornitheine decarboxylase, were also substantially suppressed by all three herbal extracts (Fig. 6).

Effects of ethanol extracts of I'm-Yunity, I'm-Yunity-plus, and Danshen on expression of breast cancer and estrogen receptor signaling genes using cDNA array analysis. We further analyzed gene responses using human breast cancer/estrogen receptor signaling cDNA arrays. The GEArray Q series human breast cancer/estrogen receptor signaling gene array is designed for the investigation of breast cancer-related gene regulation and estrogen receptor-dependent signal transduction. Although the estrogen receptor plays an important role in the pathogenesis of many breast cancers, a number of additional estrogen-independent signaling pathways are also associated with the disease. Many genes are known to be preferentially expressed in breast cancer cells and thus serve as useful markers of the disease. The expression of other genes defines the prognosis of breast cancer, while the expression of still other genes is associated with the response of cancer cells to chemotherapy. All of these genes as well as those involved in the estrogen-signaling pathway are included on this array (Fig. 7). 


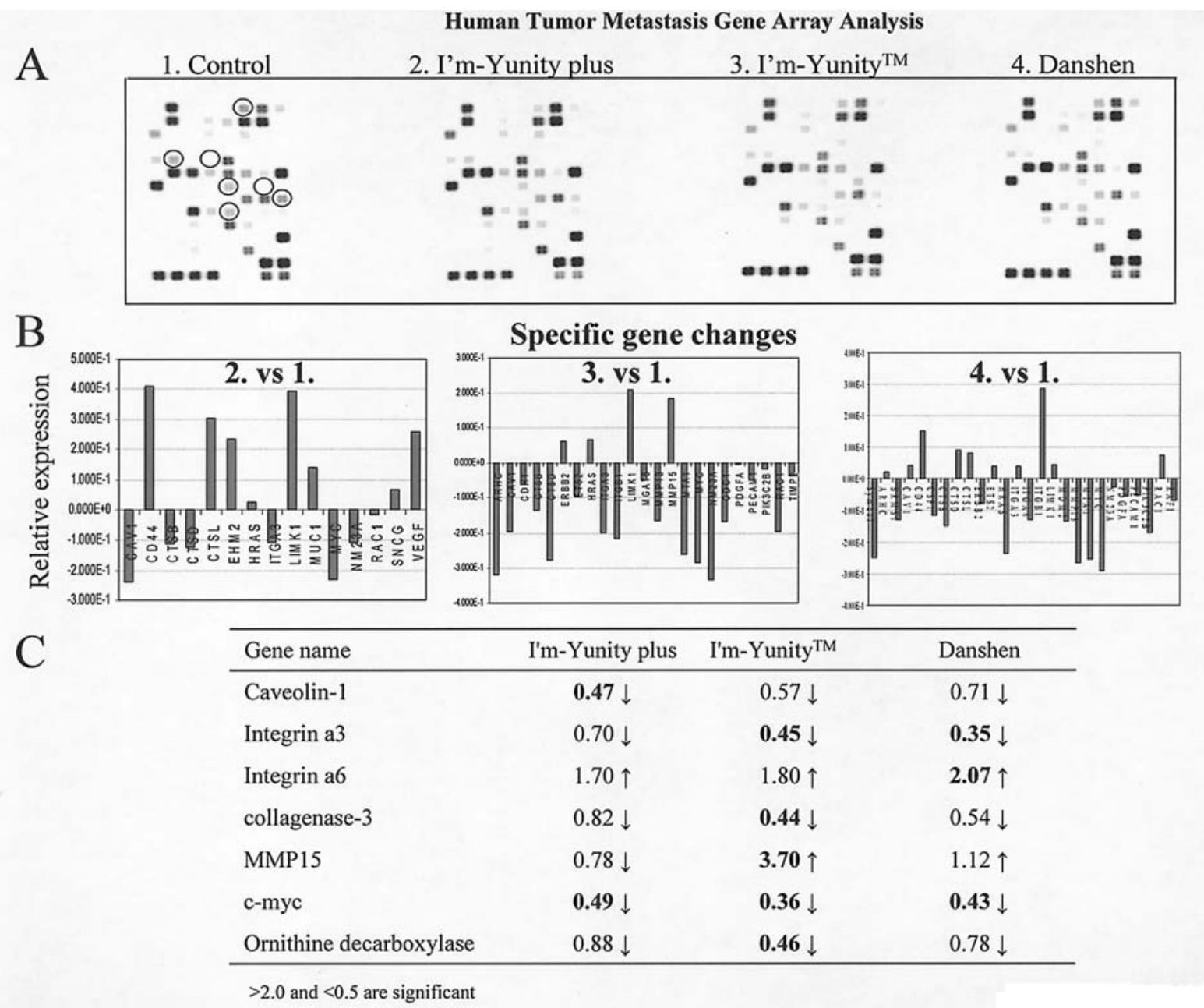

Figure 6. Identity and cellular role of human metastasis genes regulated by a 2-day treatment with $5.5 \mu 1 / \mathrm{ml}$ ethanolic extracts of I'm-Yunity, I'm-Yunity-plus, or Danshen. The GEArray Q series human tumor metastasis gene array is designed to represent 96 genes known to be involved in metastasis. Quantitative analysis of genes circled in panel A appear in panels B and C, respectively.

Again, cells treated for 2 days with ethanol extracts of I'm-Yunity, I'm-Yunity-plus, and Danshen were used in the analysis. These studies revealed a significant up-regulation of p21. Other genes whose expression increased included IL-6 and VEGF. Suppression of PR and FGF1 was also found.

\section{Discussion}

I'm-Yunity and Danshen are popular dietary supplement in the Orient, which show beneficial effects with little toxicity (34). The main active components of I'm-Yunity is a polysaccharopeptide (18-20). In the case of Danshen, several bioactive compounds have been identified (35-37). Since these bioactive agents have substantially different biochemical characteristics, we reasoned that combination of I'm-Yunity and Danshen may interact in ways that provide evidence for biological synergy. This possibility appears to be validated by our studies using human MCF-7 cells.

We observed that proliferation in MCF-7 cells was inhibited to a comparable degree by I'm-Yunity-plus and Danshen, and to a lesser degree, also by I'm-Yunity (Fig. 2A). Clonogenicity assay using these cells, however, showed Danshen to be more effective than I'm-Yunityplus and I'm-Yunity (Fig. 1). Flow cytometric analysis revealed that $7.5 \mu \mathrm{l} / \mathrm{ml}$ Danshen induced $\mathrm{G}_{1} / \mathrm{S}$ arrest while I'm-Yunity-plus elicited a prolongation of $\mathrm{G}_{1} / \mathrm{S}$ transition into $\mathrm{G}_{2} / \mathrm{M}$ phase of the cell cycle (Fig. 3). Little apoptosis was shown in MCF-7 cells treated with I'mYunity-plus or Danshen, which was strikingly different from the significant induction of apoptosis in cells treated with I'm-Yunity (Fig. 3). To investigate the biochemical basis for the observed flow cytometric results, we analyzed changes in cell cycle regulatory proteins. Only Danshen-treated MCF-7 cells showed diminished expression of cyclin D1, Rb and p53. Similarly, p65 or p50 forms of NFKB were affected by Danshen in MCF-7 cells (Fig. 5). These results contrasted with little to no observed changes in cyclin $\mathrm{D} 1, \mathrm{Rb}$ and $\mathrm{p} 53$, or either form of NFKB in MCF-7 cells treated with either I'm-Yunity or I'm-Yunityplus (Figs. 4 and 5).

Control of tumor metastasis and estrogen receptor signaling by I'm-Yunity, I'm-Yunity-plus, and Danshen: analysis using cDNA arrays. To further investigate that synergy could arise from combining Danshen with I'm-Yunity, we resorted to comparison of the expression profile of gene sets that either regulate or are involved in tumor metastasis and estrogen receptor signaling in $\mathrm{MCF}-7$ cells. To assess the relative 


\section{Human Breast Cancer and Estrogen Receptor Signaling Gene Array Analysis}

Control I'm-Yunity plus I'm-Yunity

\begin{tabular}{lccc}
\hline B Gene name & I'm-Yunity plus & I'm-Yunity & Danshen \\
\hline Cyclin D1 & $\mathbf{0 . 4 4} \downarrow$ & $1.02 \uparrow$ & $0.86 \downarrow$ \\
p21/Waf1/CIP1 & $\mathbf{3 . 7 6} \uparrow$ & $\mathbf{2 . 0 6} \uparrow$ & $\mathbf{2 . 1 5} \uparrow$ \\
FGF1 & $\mathbf{0 . 3 6} \downarrow$ & $0.81 \downarrow$ & $0.54 \downarrow$ \\
IL6 & $\mathbf{1 6 . 0 1} \uparrow$ & $\mathbf{2 . 7 5} \uparrow$ & $\mathbf{6 . 9 2} \uparrow$ \\
PR & $\mathbf{0 . 2 7} \downarrow$ & $0.95 \downarrow$ & $0.69 \downarrow$ \\
CD98 & $\mathbf{3 . 6 0} \uparrow$ & $\mathbf{2 . 3 9} \uparrow$ & $\mathbf{3 . 2 7} \uparrow$ \\
VEGF & $\mathbf{2 . 8 1} \uparrow$ & $1.29 \uparrow$ & $\mathbf{2 . 2 9} \uparrow$ \\
\hline
\end{tabular}

$>2.0$ and $<0.5$ are significant

Figure 7. Identity and cellular role of breast cancer/estrogen receptor signaling genes regulated by a 2-day treatment with $5.5 \mu 1 / \mathrm{ml}$ ethanolic extracts of I'mYunity, I'm-Yunity-plus, or Danshen. The GEArray Q series human breast cancer/estrogen receptor signaling gene array is designed for the investigation of breast cancer-related gene regulation and estrogen receptor-dependent signal transduction. Quantitative analysis of genes circled in panel A appear in panel B.

response of functionally related gene sets quickly and accurately, a cDNA array analysis was performed. Unlike classic RNA methodologies such as Northern blot analysis, studying one gene at a time, low-density arrays were selected for ease of operation and management of data; comparison of the differential hybridization outputs between control and treated MCF-7 cells enabled systematic and reliable comparison of relative levels of many mRNA transcripts simultaneously in a single experiment. Results derived from computer analysis provided semi-quantitative information that may provide clues to an understanding of complex pathways and their interactions in gene regulation that underlie hormone-dependent breast carcinogenesis and that may be targeted by I'm-Yunity, I'm-Yunity-plus, and Danshen.

By comparing the expression profile after 2 day of treatment with I'm-Yunity, I'm-Yunity-plus, and Danshen, we found that p21 was the most highly induced gene in treated MCF-7 cells. The p21 is a potent mitotic inhibitor, which inhibits cdc2 expression. Additionally, the p21 can also inhibit Rb phosphorylation by binding to cdk to effectively block the catalytic activities of cdk-cyclin complexes, e.g., cyclins A/Ecdk2, and cyclin D1/D2-cdk4. Moreover, the highly induced expression of p21 following I'm-Yunity, I'm-Yunity-plus, and Danshen treatment would ensure the growth arrest at $\mathrm{G}_{1}$. Our array analysis revealed this to be an unreported target of I'mYunity, I'm-Yunity-plus, and Danshen.

The use of gene array analysis should provide the broadest scientific framework relevant to the anti-BCa properties of
I'm-Yunity, I'm-Yunity-plus, and Danshen. Furthermore, since substantial variation in efficacy exists between different batches of I'm-Yunity, I'm-Yunity-plus, and Danshen, and methods for their standardization are as yet unavailable, the gene profile approach could also subserve as a reproducible, sensitive, and easily adaptable laboratory indicator confirming/refuting the lot-to-lot consistency status of I'm-Yunity, I'm-Yunity-plus, and Danshen, and may be used to guide the development of I'm-Yunity, I'm-Yunity-plus, and Danshen with more potent biological properties.

In summary, our results are consistent with the interpretation that interaction exists between Danshen and I'mYunity and that I'm-Yunity-Plus may be useful in the treatment of $\mathrm{BCa}$, particularly in patients with $\mathrm{ER}^{+}$status.

\section{Acknowledgements}

This work was supported in part by an unrestricted grant from the Hong Kong Healthcare Center Ltd. We extend special thanks to Professor Wen-hsien Chou, Suzhou University, for his continuing interest and unwavering support of this work.

\section{Reference}

1. Marshall E: Epidemiology. Search for a killer: focus shifts from fat to hormones. Science 259: 618-621, 1993.

2. Perera NM and Gui GP: Multi-ethnic differences in breast cancer: current concepts and future directions. Int J Cancer 106: 463-467, 2003. 
3. Rock E and De Michele A: Nutritional approaches to late toxicities of adjuvant chemotherapy in breast cancer survivors. J Nutr 133: S3785-S3793, 2003.

4. Lee MM, Lin SS, Wrensch MR, Adler SR and Eisenberg D: Alternative therapies used by women with breast cancer in four ethnic populations. J Natl Cancer Inst 92: 42-47, 2000.

5. Halicka HD, Ardelt B, Juan G, Mittleman A, Chen S, Traganos F and Darzynkiewicz Z: Apoptosis and cell cycle effects induced by extracts of the Chinese herbal preparation PC SPES. Int $\mathbf{J}$ Oncol 11: 437-448, 1997.

6. Hsieh TC, Wijeratne EK, Liang JY, Gunatilaka AL and Wu JM: Differential control of growth, cell cycle progression, and expression of NF-kappaB in human breast cancer cells MCF-7, MCF-10A, and MDA-MB-231 by ponicidin and oridonin, diterpenoids from the chinese herb Rabdosia rubescens. Biochem Biophys Res Commun 337: 224-231, 2005.

7. Whitehead N: Herbal remedies: integration into conventional medicine. Nurs Times 99: 30-33, 2003.

8. Bonakdar RA: Herbal cancer cures on the Web: noncompliance with The Dietary Supplement Health and Education Act. Fam Med 34: 522-527, 2002.

9. Kuruvilla A: Herbal formulations as pharmacotherapeutic agents. Indian J Exp Biol 40: 7-11, 2002.

10. Yuan R and Lin Y: Traditional Chinese medicine: an approach to scientific proof and clinical validation. Pharmacol Ther 86: 191-198, 2000.

11. Darzynkiewicz Z, Traganos F, Wu JM and Chen S: Chinese herbal mixture PC SPES in treatment of prostate cancer (Review). Int J Oncol 17: 729-736, 2000.

12. Hsieh TC, Lu X, Guo J, Xiong W, Kunicki J, Darzynkiewicz Z and $\mathrm{Wu}$ JM: Effects of herbal preparation Equiguard on hormoneresponsive and hormone-refractory prostate carcinoma cells: mechanistic studies. Int J Oncol 20: 681-689, 2002.

13. Hsieh TC, Lu X, Chea J and Wu JM: Prevention and management of prostate cancer using PC-SPES: a scientific perspective. J Nutr 132: S3513-S3517, 2002.

14. Sporn MB and Suh N: Chemoprevention: an essential approach to controlling cancer. Nat Rev Cancer 2: 537-543, 2002.

15. Sporn MB and Suh N: Chemoprevention of cancer. Carcinogenesis 21: 525-530, 2000

16. Sporn MB and Newton DL: Chemoprevention of cancer with retinoids. Fed Proc 38: 2528-2534, 1979.

17. Sporn MB, Dunlop NM, Newton DL and Smith JM: Prevention of chemical carcinogenesis by vitamin A and its synthetic analogs (retinoids). Fed Proc 35: 1332-1338, 1976.

18. Wasser SP and Weis AL: Therapeutic effects of substances occurring in higher Basidiomycetes mushrooms: a modern perspective. Crit Rev Immunol 19: 65-96, 1999.

19. Chu KK, Ho SS and Chow AH: Coriolus versicolor: a medicinal mushroom with promising immunotherapeutic values. J Clin Pharmacol 42: 976-984, 2002.

20. Cui J and Chisti Y: Polysaccharopeptides of Coriolus versicolor: physiological activity, uses, and production. Biotechnol Adv 21: 109-122, 2003.

21. Kidd PM: The use of mushroom glucans and proteoglycans in cancer treatment. Altern Med Rev 5: 4-27, 2000.

22. Chen XG, Li Y, Yan CH, Li LN and Han R: Cancer chemopreventive activities of S-3-1, a synthetic derivative of danshinone. J Asian Nat Prod Res 3: 63-75, 2001

23. Liu J, Yang CF, Wasser S, Shen HM, Tan CE and Ong CN: Protection of salvia miltiorrhiza against aflatoxin-B1-induced hepatocarcinogenesis in Fischer 344 rats dual mechanisms involved. Life Sci 69: 309-326, 2001.
24. Hsieh TC and Wu JM: Differential effects on growth, cell cycle arrest, and induction of apoptosis by resveratrol in human prostate cancer cell lines. Exp Cell Res 249: 109-115, 1999.

25. Hsieh TC and Wu JM: Cell growth and gene modulatory activities of Yunzhi (Windsor Wunxi) from mushroom Trametes versicolor in androgen-dependent and androgen-insensitive human prostate cancer cells. Int J Oncol 18: 81-88, 2001.

26. Hsieh TC, Wang Z, Hamby CV and Wu JM: Inhibition of melanoma cell proliferation by resveratrol is correlated with upregulation of quinone reductase 2 and p53. Biochem Biophys Res Commun 334: 223-230, 2005.

27. Fu Y, Hsieh TC, Guo J, Kunicki J, Lee MY, Darzynkiewicz Z and Wu JM: Licochalcone-A, a novel flavonoid isolated from licorice root (Glycyrrhiza glabra), causes $\mathrm{G} 2$ and late-G1 arrests in androgen-independent PC-3 prostate cancer cells. Biochem Biophys Res Commun 322: 263-270, 2004.

28. Di Pietrantonio AM, Hsieh TC, Olson SC and Wu JM: Regulation of G1/S transition and induction of apoptosis in HL-60 leukemia cells by fenretinide (4HPR). Int J Cancer 78: 53-61, 1998.

29. Hsieh TC, Kunicki J, Darzynkiewicz Z and Wu JM: Effects of extracts of Coriolus versicolor (I'm-Yunity) on cell-cycle progression and expression of interleukins- 1 beta, -6 , and -8 in promyelocytic HL-60 leukemic cells and mitogenically stimulated and nonstimulated human lymphocytes. J Altern Complement Med 8: 591-602, 2002.

30. Hsieh TC, Burfeind P, Laud K, Backer JM, Traganos F, Darzynkiewicz Z and Wu JM: Cell cycle effects and control of gene expression by resveratrol in human breast carcinoma cell lines with different metastatic potentials. Int J Oncol 15: 245-252, 1999.

31. Hsieh TC, Juan G, Darzynkiewicz Z and Wu JM: Resveratrol increases nitric oxide synthase, induces accumulation of p53 and $\mathrm{p} 21(\mathrm{WAF} 1 / \mathrm{CIP} 1$ ), and suppresses cultured bovine pulmonary artery endothelial cell proliferation by perturbing progression through S and G2. Cancer Res 59: 2596-2601, 1999.

32. Tsai SY, Ardelt B, Hsieh TC, Darzynkiewicz Z, Shogen K and Wu JM: Treatment of Jurkat acute T-lymphocytic leukemia cells by onconase (Ranpirnase) is accompanied by an altered nucleocytoplasmic distribution and reduced expression of transcription factor NF-kappaB. Int J Oncol 25: 1745-1752, 2004.

33. Baeuerle PA and Baltimore D: NF-kappa B: ten years after. Cell 87: 13-20, 1996.

34. Leung CH, Grill SP, Lam W, Han QB, Sun HD and Cheng YC: Novel mechanism of inhibition of nuclear factor-kappa B DNAbinding activity by diterpenoids isolated from Isodon rubescens. Mol Pharmacol 68: 286-297, 2005.

35. Wu CT, Mulabagal V, Nalawade SM, Chen CL, Yang TF and Tsay HS: Isolation and quantitative analysis of cryptotanshinone, an active quinoid diterpene formed in callus of Salvia miltiorrhiza BUNGE. Biol Pharm Bull 26: 845-848, 2003.

36. Hu P, Luo GA, Zhao ZZ and Jiang ZH: Quantitative determination of four diterpenoids in Radix Salviae Miltiorrhizae using LC-MS-MS. Chem Pharm Bull 53: 705-709, 2005.

37. Hu P, Liang QL, Luo GA, Zhao ZZ and Jiang ZH: Multicomponent HPLC fingerprinting of Radix Salviae Miltiorrhizae and its LC-MS-MS identification. Chem Pharm Bull 53: 677$683,2005$. 